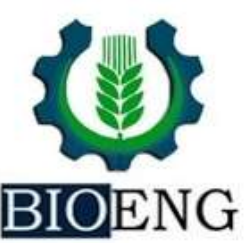

\title{
APLICAÇÃO DO DMAIC PARA SOLUÇÃO DE LATAS AMASSADAS EM PROCESSOS DE UMA FABRICA DE LEITE EM PÓ
}

\author{
S. da S. Rêgo; W. A. P. Syed e G. A. Prates"
}

\section{UNESP- Univ Estadual Paulista, Campus de Itapeva, SP, Brasil}

Article history: Received 27 May 2015; Received in revised form 13 June 2015; Accepted 20 June 2015; Available online 30 June 2015.

\section{RESUMO}

Problemas com latas enferrujadas, amassadas ou estufadas, podem levar a doenças como o botulismo por conterem bactérias que causam infecções alimentares. Na empresa estudada, localizada no interior de São Paulo, como em todas do segmento, o leite em pó é embalado, majoritariamente por latas de alumínio que são revestidas internamente com vernizes e outros materiais visando proteger o leite de metais provenientes do alumínio. Por conseguinte, o objetivo do estudo era a resolução do problema das latas amassadas que para tanto foi utilizado como metodologia um estudo de caso com abordagem quantitativa através do método DMAIC desenvolvida pelo Seis Sigma. Foi realizado um levantamento sobre o descarte de latas na empresa verificando-se uma perda média e, a partir desse histórico, calculada uma meta de perda. Com a meta definida calculou-se o saving anual do projeto. Durante a aplicação do DMAIC, ficou constatado que a maior taxa de perda ocorria no transporte entre a fábrica de latas e a fábrica que envasa o leite. Foram realizadas diversas ações para solucionar problemas que resultavam em latas amassadas e, nos dois primeiros meses da fase control, constatou-se perdas menores que a meta calculada, resultando em uma economia. Observou-se que o transporte entre as duas fábricas ainda é impactante no descarte de latas.

Palavras-chave: DMAIC, Perda de Latas, Ferramentas da Qualidade, Seis sigma

\section{DMAIC METHODS FOR CANS CRUSHED PROBLEM SOLLUTION IN A MILK POWDER INDUSTRY}

\begin{abstract}
Problems with rusty cans, crushed or stewed, can lead to diseases like Botulism due to contain bacteria that cause foodborne illness. This study was conducted at the milk powder company in São Paulo State, Brazil. On every segment, the milk powder is packed, mostly by aluminum cans are internally coated with varnishes and other materials to protect the metal milk taste-taken over from aluminum. Therefore, the study aim was to solve the problem of cans crushed that for both methodology was used as a case study with a quantitative approach through the DMAIC method developed by Six SGMA. It conducted a observation on disposal cans at the company verifying an average loss and, from that historic data, calculated a loss of goal. A target set was calculated the annual saving project. During the application of DMAIC,
\end{abstract}


it was found that the loss highest rate were occurring in transport cans between the factory and the factory that fills milk. Several actions were taken to address problems that resulted in dented cans and in the first two months of control phase, there was lower losses than the calculated goal, resulting in savings. It was noted that the transport between the two plants is still affecting the discard canisters.

Keywords: DMAIC, Can loss, Quality Management Tools, Six sigma

\section{INTRODUÇÃO}

Segundo Machado \& Cassoli (2010) o leite em pó é um dos produtos lácteos mais exportados pelo Brasil, o qual até a década de 80 era considerado um grande importador de leite em pó. Entretanto, com o passar dos anos se tornou um grande exportador chegando a ocupar a oitava posição no ranking mundial de exportação de leite em pó.

O leite em pó, assim como outros produtos, é embalado por latas metálicas formadas por folhas de flandres. O uso desse tipo de embalagem visa à proteção do produto, resultando em maior vida útil ao mesmo (ABRE, 2004). As latas metálicas são protegidas com vernizes e outros materiais orgânicos para evitar a contaminação do produto que embalam por metais, mas quando as latas são amassadas, essa proteção fica comprometida (DANTAS, 2008). Segundo Bressan et al (1999) problemas com latas enferrujadas, amassadas ou estufadas, podem levar a doenças como o botulismo por conterem bactérias que causam infecções alimentares.

Além dos problemas de contaminação do leite em pó, o problema de latas amassadas pode ser considerado como uma perda industrial. Os principais focos para redução de custos no setor de

\section{Método DMAIC}

A seguir são descritas as fases do DMAIC:

\section{Define}

Segundo Amr \& Khalifa (2014) o primeiro passo dessa fase é encontrar o processo que pode ser melhorado para atender a alguma exigência ou necessidade dos clientes. A seguir devese considerar, qual é o histórico do manufatura são as perdas geradas pela ineficiência dos processos e as perdas por defeitos, como o de latas amassadas. Para solução desses problemas, buscamse alternativas eficientes que sejam lucrativas ou tenham apelo ambiental ou social. Os Seis Sigmas surgiram como alternativa para suprir essas carências, pois através do método Define, Measure, Analyze, Improve e Control (DMAIC), é possível resolver problemas de forma metódica alcançando, entre outros objetivos, o retorno financeiro.

Segundo Chakrabortty et al. (2013); Selvi \& Majumdar (2014) hoje em muitas indústrias de transformação lideres começaram a implantar o Six Sigma e conceitos de manufatura enxuta para aumentar a sua produtividade, bem como a qualidade dos produtos. A principal característica dos Seis Sigmas é a busca por melhores desempenhos no processo, independente do valor obtido ao final do projeto (GUPTA, 2013).

Este trabalho tem como objetivo demonstrar um potencial de redução da ocorrência de latas amassadas no setor de embasamento de leite em pó de uma indústria de alimentos através da metodologia DMAIC em um projeto White Belt. problema; qual a meta geral a ser definida; quais as perdas atuais e ganhos do projeto; qual é a equipe formada para conduzir o projeto; se o contrato do projeto foi realizado e; por último; qual será o cronograma de trabalho 
(MATATHIL; GANAPATHI \&
RAMACHANDRAN, 2012).

\section{Measure}

Nesta fase, deverão ser abordados os seguintes pontos: se o problema está específico; quais os critérios que serão utilizados para desdobrar o problema; verificar se há dados confiáveis; verificar quais são os problemas prioritários identificados após a estratificação dos dados; verificar se o "follow-up" do plano de ação de gestão do projeto foi realizado e por último, deve-se verificar se foi realizada uma avaliação da equipe do projeto (NICOLI \& NIKOLIC, 2013).

\section{Analyze}

Segundo Kaushik et al (2012), Tjahjono et al. (2010) nessa etapa devem-se verificar os seguintes pontos: se foram restauradas as condições básicas de operação; quais as causas influentes do problema prioritário identificadas no brainstorming. Como foram organizadas e agrupadas no diagrama de causa e efeito; quais foram as causas raízes identificadas no teste dos "5 Porquês"; se

\section{DMAIC em indústrias de alimentos}

Hung \& Sung (2011) explora a forma como uma empresa de alimentos em Taiwan pode usar uma abordagem sistemática e disciplinada para se mover em direção à meta de nível de qualidade Six Sigma. As fases DMAIC são utilizados para diminuir a taxa de defeito de pequenos bolos de creme em $70 \%$ da linha de base para a sua titularidade. No início do projeto, a taxa de defeito foi $0,45 \%$ (base), e depois das ações de melhoria foram implementadas durante um período de seis meses, este número caiu para menos de $0,141 \%$ (meta). Os fatores de sucesso críticos para projetos Seis Sigma, especialmente aqueles na indústria de alimentos, são discutidos na conclusão deste trabalho.

Para Zhen (2011), a segurança alimentar é uma disciplina científica que descreve a manipulação, preparação e armazenamento de alimentos de forma a o follow-up do plano de ação e gestão do projeto foi realizado e; por fim, se foi realizada a avaliação da equipe do projeto (VAN DER POL, 2011).

\section{Improve}

Deverão ser explanadas as soluções propostas para eliminar as causas raízes do problema. É necessário reavaliar o plano de ação; discutindo se houve a necessidade de mudanças, verificar como foi sua execução e garantir que todos os membros da equipe estejam envolvidos (ESTORILIO \& AMITRAMO, 2013).

\section{Control}

Para realizar esse controle, os pontos a serem verificados são: se a meta foi alcançada, quais padrões foram alterados; se as melhorias oriundas do projeto foram registradas; se os funcionários foram treinados para manter o novo padrão; qual o plano de monitoramento para manter o desempenho; quais serão os ganhos efetivos do processo (MAST \& LOKKERBOL, 2012)

evitar doenças transmitidas por alimentos. Sua pesquisa apresentou a aplicação de higiene industrial (IH), aspectos no sistema de segurança alimentar e ferramentas Lean Seis Sigma no processamento salmão congelado. A utilização Modo de Análise de Efeito (FMEA) e a implementação de um plano de Análise de Perigos e Pontos Críticos de Controle (HACCP) levou à minimização de contaminação e da probabilidade do perigo biológico. A eficiência do ciclo do processo (PCE) foi melhorada de $5,02 \%$ a $17,46 \%$, devido à implementação de práticas Lean. Os dados demonstraram que ferramentas Lean Seis Sigma pode contribuir para a implementação do sistema de segurança alimentar para minimizar o risco, melhorar a produtividade e qualidade dos produtos e reduzir o desperdício de tempo e desnecessário. 


\section{MATERIAIS E MÉTODOS}

O estudo de caso foi realizado em uma indústria alimentícia localizada no estado de São Paulo. A perda de latas corresponde a grande parte da perda de material e gera reclamações nos centros de distribuição além de ocasionar pequenas paradas na linha de produção. O projeto é definido pela política da empresa como "White Belt" (Faixa Branca), um projeto DMAIC de baixa complexidade, pois utiliza ferramentas

\section{RESULTADOS}

\section{Escolha do problema}

Foi realizado um levantamento da perda de material em toda empresa e constatado que as maiores perdas são as de latas amassadas na fábrica de envasamento de leite em pó, que correspondiam a $11,78 \%$ da perda de material como um todo.

\section{Histórico do problema}

A empresa utiliza latas de quatrocentos gramas e de oitocentos gramas nos seus processos. As latas de quatrocentos gramas são responsáveis por $75,23 \%$ das perdas de latas. Foi possível visualizar uma perda média anual de latas de quatrocentos gramas de $1,48 \%$ no período de abril de 2012 a março de 2013. A maior perda de latas foi em novembro de 2012, com 1,99\%; a menor, foi em abril de 2012, com 0,72\%.

A meta foi calculada levando-se em conta os seguintes parâmetros:

- Média: a média aritmética da quantidade de latas coletadas pelo número de dias de coleta.

- Benchmark: é um valor de referência mundial do melhor resultado já alcançado para o indicador.

- Recuperação da Lacuna: porcentagem que deve ser reduzida.

A partir dos doze meses de coleta foi realizado um gráfico sequencial representando as porcentagens de perdas. Foi recalculada a média de perdas por 4. Planejamento da coleta de dados básicas e apresenta pequeno impacto. Seguindo a metodologia de aplicação do DMAIC foram realizadas as cinco etapas com aplicação de ferramentas da qualidade pertinentes a cada fase. Foram impressas folhas de verificação para auxiliar no levantamento dos dados, e para elaboração dos gráficos e diagramas, foi utilizado o programa excel.

latas amassadas obtendo-se o valor de $1,64 \%$ e um Benchmark de $1,32 \%$ de latas amassadas, assim, foi calculada a lacuna e chegou-se ao valor de $0.32 \%$. Foi estipulado pela gerência uma recuperação da lacuna de $70 \%$. Destarte, foi calculada a meta chegando-se ao valor de $1,42 \%$ de redução de latas amassadas, ou seja, uma redução de $13,49 \%$ em relação a média de $1,64 \%$.

\section{Cálculo do "saving" anual}

O saving anual se refere ao valor que a empresa economizara em um ano caso a meta seja atingida. Os parâmetros considerados foram os seguintes: Foi levantado junto ao setor administrativo qual será a quantidade de latas consumidas durante a implantação do projeto e foi estipulado que a quantidade será de 30.816.817 latas. Essa quantidade foi definida como "Consumo".

Sabendo que a média de perda de latas é de $1,64 \%$ calculou-se uma perda de 505.396 latas. Com a meta traçada de $1,42 \%$, calculou-se uma perda de 437.599 latas. O preço de cada lata de quatrocentos gramas é de $\mathrm{R} \$ 0,30$, sendo assim, calculou-se um saving anual de $\mathrm{R} \$$ 20.001,00, equivalente a US\$ $8.776,44$. 
Foi realizado um plano de coleta utilizando a ferramenta $5 \mathrm{~W} 1 \mathrm{H}$, conforme Quadro 1.

Quadro 1 - Plano de coleta de dado

\begin{tabular}{|l|l|}
\hline \multicolumn{2}{|c|}{ Plano de Coleta de Dados $(5 \mathrm{~W} 1 \mathrm{H})$} \\
\hline Quais dados coletar? & Latas amassadas \\
\hline Qual a unidade de medida ? & Unidades de latas \\
\hline Onde coletar os dados & Latoaria e pontos da fábrica \\
\hline Quando coletar? & Semana 15 \\
\hline Como coletar os dados? & Coletar 2 latas a cada 1 hora \\
\hline Por que coletar esses dados? & $\begin{array}{l}\text { Para definição da etapa priorizada do } \\
\text { projeto }\end{array}$ \\
\hline Quem realizará a coleta de dados? & Operadores da linha \\
\hline
\end{tabular}

\section{Definição dos pontos de coleta}

Os cinco pontos de coleta selecionados foram os seguintes:

- Paletizador: grande quantidade de choque nas latas e interferência dos operadores no manuseio.

- Depaletizador: processo sofria iguais interferências do paletizador, são processos semelhantes.

- Recravadeira: equipamento tem bastante contato com as latas e recebe grande volume.

- Gaseadoras: as latas recebem oxigênio aumentando sua pressão interna.

6. Avaliação da confiabilidade das informações no processo para os fatores de estratificação definidos.

Os dados foram coletados em 10 dias de produção normal. Foram

- Aplicador de tampas: este equipamento pressiona as latas durante a aplicação das tampas.

No aplicador de medidas e no raio $x$, não há contato direto com as latas; portanto, foram descartados como pontos de coleta. Os equipamentos aplicador de tampas $e$ encaixotadeira foram selecionados como um só ponto de coleta devido à proximidade entre estes e à velocidade do processo que inviabilizaria o operador fazer a coleta separadamente.

coletadas latas amassadas em nove etapas do processo, verificando-se que $90,10 \%$ das latas amassadas formavamse no depaletizador, demonstrado na figura 1 a seguir.

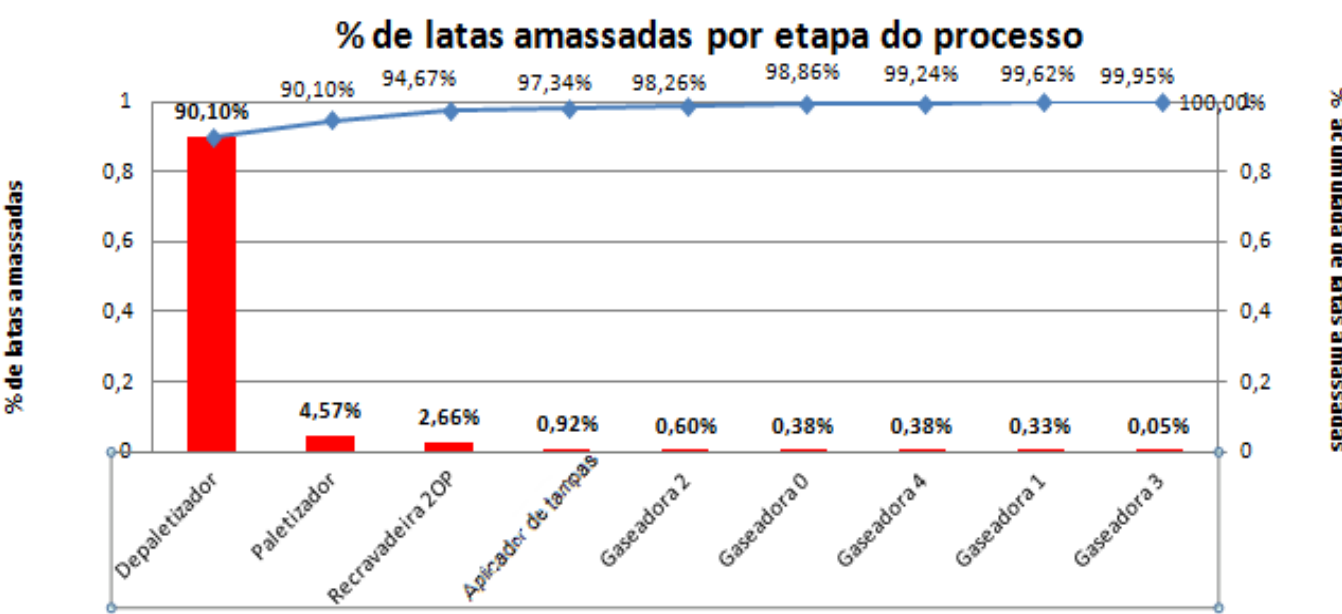

Figura 1- Gráfico da porcentagem de latas amassadas por etapa do processo. 
7. Problemas prioritários identificados após a estratificação dos dados

Através da ferramenta $5 \mathrm{~W} 1 \mathrm{H}$, foram respondidas algumas questões que possibilitaram observar a ocorrência de um elevado percentual de perda de latas na fábrica. Latas chegavam amassadas no depaletizador $\mathrm{e}$ por isso eram descartadas. $\mathrm{O}$ descarte ocorria aleatoriamente, ao longo do período de produção no transporte de latas e poderia estar relacionado com a habilidade operacional.

\section{Analyze}

\section{Definição da etapa prioritária}

Com o levantamento de dados nos pontos de coleta pôde ser observada uma diferença na quantidade de latas amassadas entre $\mathrm{o}$ paletizador $\mathrm{e} \mathrm{o}$ depaletizador. Verificou-se que o problema estava no transporte e não nos equipamentos, portanto a restauração das condições básicas não se aplicou a esta etapa.

\section{Causas influentes do problema prioritário identificadas no Brainstorming}

A equipe realizou um brainstorming para levantar as causas do amassamento de latas durante $o$ transporte entre $\mathrm{o}$ paletizador $\mathrm{e} o$ depaletizador. Estas foram agrupadas em 4Ms (Medida, Método, Meio ambiente e Matéria prima) Foram levantadas seis possíveis causas para o problema:

Pressão do strech desajustada: o strech não estava totalmente justo às latas, com isso as latas ficavam mais soltas movimentando-se durante $\mathrm{o}$ transporte como mostra a figura 2 .

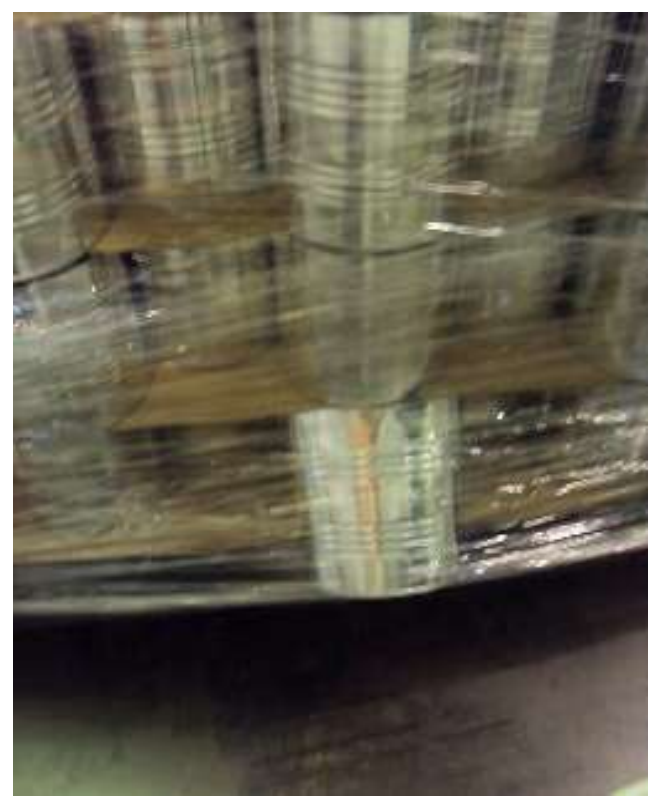

Figura 2 - Strech desajustado

Habilidade do operador que abastece e descarrega o caminhão:

Falta de strech na base do pallet: a base é a área onde as latas sofriam as maiores pressões por suportarem o peso de outras latas, sendo assim o strech passado nessa área poderia não estar sendo suficiente como demonstra a figura 3. 


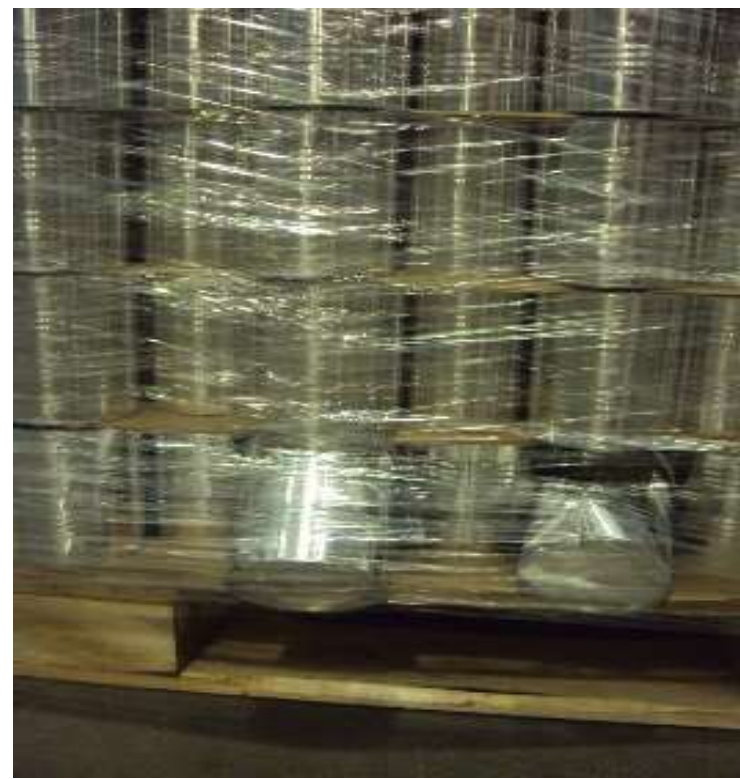

Figura 3-Strech na base do pallet

Arranjo das latas no pallet: o arranjo das latas poderia não ser o ideal, pois as latas mais externas ficavam muito expostas.

Empilhamento dos pallets: poderia ser que as latas estivessem sendo empilhas de forma inadequada, com excesso de latas e com falta de alinhamento entre as camadas.

Material de que separa as camadas de latas no pallet: as camadas de latas no pallet eram separadas por folhas de papelão do tamanho da área que as latas ocupavam no pallet; assim, com pequenas movimentações, as extremidades das latas ficam desprotegidas.

\section{Causas raízes identificadas no Teste dos "5 Porquês"}

Todas as causas foram consideradas como causas potenciais e o arranjo das latas no pallet foi considerado como causa raiz, pois caso as latas fossem arranjadas de forma a não ficarem expostas nas extremidades, poderia ser que não ocorresse grande parte das causas.

\section{Improve}

\section{Soluções propostas para eliminar as causas potenciais}

Foram propostas 9 prováveis soluções para as causas enumeradas no teste dos 5 porquês:

1. Consertar o motor do strech: verificou-se que o motor do strech se encontrava quebrado, com isso o strech estava sendo colocado de forma manual, ocasionando uma falta pressão no mesmo deixando as latas soltas.

2. Padronizar a pressão do strech: verificou-se uma falta de padrão na pressão do strech; manualmente, não havia controle, e mesmo com a utilização do equipamento, verificouse que os operadores não tinham conhecimento sobre um padrão para se utilizar.

3. Dar treinamento de conscientização dos impactos da lata amassada no processo: verificou-se que a perda de latas havia se tornado comum na fábrica e era encarada pelos funcionários como uma situação aceitável, com isso ocorria muita displicência em algumas operações.

4. Contratar mais empilhadeiristas: observou-se que o volume de latas a serem transportadas era muito grande, com isso os empilhadeiristas operavam de forma muito veloz, comprometendo a qualidade do transporte. 
5. Dar treinamento de boas práticas de transporte: verificou-se a necessidade de fornecer um treinamento específico para os empilhadeiristas, pois alguns operavam as empilhadeiras de forma displicente causando impactos nas latas.

6. Aumentar o número de voltas de strech na base do pallet: como demonstrado na figura 3 , observou-se que na base do pallet as latas sofriam maiores impactos e com isso se desprendiam facilmente do stretch. Com um aumento no número de voltas de stretch, as latas não se desprenderiam tão facilmente.

7. Ajustado o paletizador para centralizar as latas no pallet: observou-se que as latas ficavam muito expostas nas extremidades do pallet. Para que isso não ocorresse, uma solução seria colocar as latas apenas no centro dos pallets, analisar a demanda para que o volume de latas no depósito diminua e permita o máximo de dois pallets empilhados: caso o número de pallets empilhados diminuísse, as latas sofreriam menor pressão na base e o transporte dos empilhadeiristas seria menos impactante.

8. Trocar o material das divisórias por um mais resistente: o material utilizado era um papelão que absorvia pouco impacto. Caso esse papelão fosse trocado por um de espessura maior, ele transmitiria menos impactos às latas.

9. Testar divisórias maiores para verificar se as latas ficariam melhor arranjadas: as divisórias estavam ocupando exatamente a área que as latas ocupavam no pallet. Para proteger as latas mais externas, a troca da divisória por uma um pouco maior seria uma solução, pois assim, formariam bordas com a sobra da divisória.

\section{Elaboração do plano de ação}

Observou-se que quatro etapas acarretariam custos como a troca de material que separa as camadas de latas no pallet por um papelão de espessura maior, de custo $\mathrm{R} \$ 8.000,00$, o rearranjo das latas no pallet que necessitaria de um investimento de R\$ 15.000,00; a contratação de empilhadeiristas custando $\mathrm{R} \$ 1.500,00$ por mês, cada funcionário e a troca das divisórias horizontais que custariam $\mathrm{R} \$ 17.000,00$.

\section{Execução do plano de ação}

Foi possível realizar sete ações das nove sugeridas no plano de ação: Não foi possível mudar o arranjo das latas no pallet, pois o equipamento que arranja as latas é muito antigo e não aceita outra configuração. A equipe de manutenção calibrou o sensor de pressão do strech filme. Foram realizados testes na máquina de strech filme e chegou-se a uma pressão ideal que foi definida como padrão. A equipe de manutenção ajustou a máquina de strech para que ela funcionasse no modo automático, otimizando o processo. Com a pressão do strech regulada economizou-se material suficiente para dar mais duas voltas na base de cada pallet. Os empilhadeiristas receberam um treinamento sobre como transportar as latas de maneira adequada. Foram trocadas as divisórias por um papelão mais espesso que absorvia mais impactos. Foram trocadas as divisórias horizontais por divisórias maiores, sendo assim, as latas mais externas ficaram protegidas por uma borda. Considerou-se inviável a contratação de mais empilhadeiristas, pois acarretaria em um alto custo.

\section{Verificar se a meta foi alcançada.}

No mês de setembro, a variação de uso foi a perda de $0,9 \%$, enquanto no mês de outubro a variação foi de $0,85 \%$, que foi proporcionalmente ao resultado encontrado por (HUNG \& SUNG, 2011).

\section{Padrões alterados}

Foi criado um plano de manutenção da máquina strech filme após a criação de padrão visual da pressão do strech. Nesse padrão, foram 
estabelecidos limites ideais de pressão que os operadores poderiam trabalhar. As áreas verdes são as faixas de pressão que os operadores podem trabalhar, já as áreas vermelhas são faixas nas quais as pressões não são adequadas para um correto ajuste do strech. Todos os operadores da máquina strech filme foram treinados no padrão visual de pressão. Foram treinados, também, todos os empilhadeiristas que faziam o transporte das latas entre o paletizador e o depaletizador.

\section{Ganhos efetivos do processo}

Nos dois primeiros meses da fase control, foram utilizadas 7.000.000 latas sendo 3.000.000 no mês de Setembro e 4.000.000 no mês de Outubro. O histórico de perdas era de $1,64 \%$ (essa porcentagem de perda em setembro representaria 49.200 latas perdidas e 65.600 latas perdidas em outubro). Em setembro, a variação de uso foi de $0,9 \%$,

\section{DISCUSSÃO}

Kaushik et al (2012), Tjahjono et al. (2010) citam que metodologia Seis Sigmas tem como diferencial o retorno financeiro, enquanto Prates (2004) discorre que a identificação de projetos Seis Sigma permite à organização reconhecer como os seus processos afetam sua lucratividade, e permite definir quais desses processos são críticos para o negócio da empresa. Como descrito por Hung \& Sung (2011), ocorreram economias pois com a meta traçada no trabalho estimou-se um saving anual de aproximadamente $\mathrm{R} \$$ 20.000,00. Nos dois primeiros dois meses de implantação do projeto, verificou-se que $o$ saving foi de $\mathrm{R} \$$ 16.140,00.

Corroborando com Selvi \& Majumdar (2014) é necessário uma mudança na cultura organizacional começando pela conscientização e o que representa um total de 27.000 latas perdidas. Em outubro a variação de uso foi de $0,85 \%$ representando um total de 34.000 latas perdidas. Sabendo-se que o preço unitário de cada lata é de $\mathrm{R} \$ 0,30$, calculou-se um saving de $\mathrm{R} \$ 6.660,00$ (US\$ 2.922,41) em Setembro e R\$ 9.480,00 (US\$ 4.159,82) em outubro; ou seja, nos dois primeiros meses o saving calculado foi de $\mathrm{R} \$ 16.140,00$ (US\$ 7.082,23).

Existe um grande número de movimentação da linha até a chegada da lata na fábrica de envasamento de leite, causando ainda o amassamento das latas. Um transporte direto das latas sem o contato com empilhadeiras e armazenamento poderia diminuir o problema. Para fazer esse transporte pode-se estudar a viabilidade de aplicar um transportador de correias entre as duas fábricas, uma vez que causa menos impactos do que as operações com empilhadeiras.

comprometimento da gerência. Observou-se durante a coleta de dados e a aplicação dos treinamentos que a participação e compreensão do projeto da gerência foram determinantes para $o$ comprometimento dos demais colaboradores.

Para Amr \& Khalifa (2014) no caso de algum processo estar sendo feito de forma inadequada deve-se tomar ações corretivas para solucionar o processo dando treinamento para $\mathrm{o}$ colaborador de forma a se retomar o controle do processo. Criaram-se padrões visuais para operação da máquina de strech, para tanto foram dados treinamentos necessários para os colaboradores operarem o equipamento de forma correta. Os operadores das empilhadeiras também foram treinados para fazerem o transporte das latas de maneira segura. 


\section{CONCLUSÕES}

Como demonstrado nos resultados o potencial de redução da ocorrência de latas amassadas no setor de embasamento de leite em pó de uma indústria de alimentos através da metodologia DMAIC em um projeto White Belt,foi satisfatório e atendeu o objetivo do artigo.

Apesar de o projeto estar apenas no segundo mês da fase control, foi possível observar um saving de $\mathrm{R} \$ 16.140,00$ (US\$7.082,23), além disso, pôde-se observar que, nos dois primeiros meses, os resultados de perda foram melhores do que a meta calculada. Foram realizadas melhorias como implantação de padrões visuais na máquina de strech, treinamento dos empilhadeiristas que aprenderam como transportar as latas de forma segura e houve um trabalho de conscientização de todos os

\section{REFERÊNCIAS BIBLIOGRÁFICAS}

ABRE (Associação Brasileira de Embalagens) 2004/ 2008. 2008

AMR, NOAMAN \& KHALIFA, MOHAMED AMR. Process Increments. An Agille

Approach to Software Process

Improvement, IEEEXplore. 2014.

BRESSAN, M. C.; GALINDO, G. M.; SANTOS, M. A . O Botulismo no Homem, nos Animais e em alimentos. Lavras, 1999. 19p.

CHAKRABORTTY, RIPON KUMAR; BISWAS, TARUN KUMAR; AHMED, IRAJ. Reducing Process Variability by Using Dmaic Model: A Case Study in Bangladesh. International Journal for Quality Research 7(1) 127 - 140. 2011.

DANTAS, Silvia Tondella. Embalagens Metálicas e a sua Interação com Alimentos e Bebidas. Campinas, SP: ITAL/CETEA, 1999.

ESTORILIO, C. C. A. \& AMITRANO, F.G. . Aplicação de Seis Sigma em uma colaboradores da fábrica tencionando mostrar a importância de evitar as perdas de latas.

Como fatores limitantes, tempo escasso de implementação da fase improve não permitiu fazer uma análise mais detalhada sobre os ganhos reais do projeto e sobre eventuais problemas que poderão ocorrer ao longo da fase control, mas até o mês de outubro de 2013 foi possível observar que o transporte entre as duas fábricas ainda causa $o$ amassamento de latas, mas com menos frequência do que antes da implementação do projeto. Dessa maneira, este problema remanescente poderá ser objeto de estudo de um futuro trabalho assim como o transporte do produto acabado até o consumidor, que não foi abordado por esse projeto.

empresa de pequeno porte Produto \& Produção, vol. 14 (2) p. 01-25, 2013.

GUPTA, N. An Application of DMAIC Methodology for Increasing the Yarn Quality in Textile Industry. Journal of Mechanical and Civil Engineering (IOSR-JMCE) V. 6 (9), p. 50-65, 2013.

HUNG, HSIANG-CHIN \& SUNG, MING-HSIEN. Applying six sigma to manufacturing processes in the food industry to reduce quality cost. Scientific Research and Essays Vol. 6(3), pp. 580591, 4 February, 2011

KAUSHIK, P.; KHANDUJA, D.; MITTAL, K.; JAGLAN, P. A case study; Application of Six Sigma methodology in a small and medium-sized manufacturing enterprise. TQM Journal. v.24, n.1, p. 4-16, 2012.

MACHADO, P. F.; CASSOLI, L. D. Qualidade do leite no Brasil vs outros países produtores e exportadores. 2010. Palestra apresentada no $1^{\circ}$ Fórum DPA 
sobre Competitividade dos Lácteos Brasileiros, São Paulo, 2010.

MAST, J.; LOKKERBOL, J. An analysis of the Six Sigma DMAIC method from the perspective of problem solving. International Journal Production Economics. v.139, n.2, p. 604-614, 2012.

MATATHIL , AFZAL; GANAPATHI, K. N. \& RAMACHANDRAN, KALATHIL . Reduction of Scrap in an Electronic Assembly Line Using DMAIC Approach. SASTECH Journal V. 11(2), 2012.

NIKOLI. S. , NIKOLIC, I. . LEAN. Six Sigma in Food Industry . International Journal of Basic \& Applied Sciences IJBAS-IJENS V. 13(6), 2013.

PRATES, Glaucia Aparecida. Artigo. In: CONGRESSO VIRTUAL BRASILEIRO DE ADMISTRAÇÃO, 2004, Ribeirão Preto. GESTÃO DA QUALIDADE : SEIS SIGMA NA 3M DO BRASIL. Ribeirão Preto: Anais, 2004. 11p.

SELVI,K; MAJUMDAR, R. Six SigmaOverview of DMAIC and DMADV International Journal of Innovative Science and Modern Engineering (IJISME) V 2( 5) , 2014.

TJAHJONO, B.; BALL, P.; VITANOV, V.; SCORZAFAVE, C.; NOGUEIRA, J.; CALLEJA, J.; MINGUET, M.; NARASIMHA, L.; RIVAS, A.; SRIVASTAVA, A.; SRIVASTAVA, S.; YADAV, A. Six Sigma: a literature review. International Journal of Lean Six Sigma. v.1, n.3, p.216-233, 2010.

VAN DER POL, R. B. Aplicação do método DMAIC para redução da ocorrência de acidentes ferroviários, Juiz de Fora - 2011.

ZHEN, Ying. Food safety and lean Six sigma model. 2011.64 p. Dissertação (Mestrado em Ciência da segurança). University of Central Missouri. 2011. 